\title{
The CLEO III Upgrade
}

\author{
Thomas Coan ${ }^{1}$ \\ Physics Department, Southern Methodist University, Dallas, TX 75275, USA
}

\begin{abstract}
The CLEO detector at the Cornell Electron Storage Ring (CESR) is completing a major upgrade that significantly extends its physics reach in the study of heavy quarks and leptons. The major elements of this detector upgrade are the installation of a "fast" ring imaging Cherenkov detector, a new central drift chamber and a new barrel-style silicon strip vertex detector. Trigger and data acquisition systems are also upgraded. We summarize the status of these detector subsystems as well as the parallel CESR upgrade.
\end{abstract}

Invited talk at "The Seventh International Conference on Instrumenation for Colliding Beam Physics," Hamamatsu, Japan, Nov. 15-19, 1999.

$\overline{1}$ Supported by the U.S. Department of Energy 


\section{Introduction}

The CLEO physics program is a broad effort that emphasizes the study of $b$ and $c$ quark decays, $\tau$ lepton decays and two photon mediated reactions. Data are collected at or slightly below the $\Upsilon(4 S)$ resonance $(E \simeq 5.3 \mathrm{GeV})$ produced by $e^{+} e^{-}$collisions at the symmetric, one ring Cornell Electron Storage Ring (CESR).

Important elements of the CLEO physics program include studies of rare B decays, determination of CKM matrix elements, and possible observation of time-independent $\mathrm{CP}$ violation. Examples include a measurement of the ratio of CKM matrix elements $V_{t d} / V_{t s}$ by measuring $\Gamma(B \rightarrow \rho \gamma) / \Gamma\left(B \rightarrow K^{\star} \gamma\right)$, a measurement of $V_{u b}$ by measuring $\Gamma(B \rightarrow \rho(\omega) l \nu)$, and possible observation of $C P$ violation in $B \rightarrow K \pi$ decays. These examples, and others, involve rare reactions whose final states include pions and kaons. Large integrated luminosity and efficient charged hadron identification are necessary to reduce backgrounds, as well as statistical and systematic errors.

The CLEO III upgrade comprises two major efforts: an enhanced CLEO detector and an upgraded CESR. To substantially enlarge data sets, CESR is modified to boost its instantaneous luminosity to $L \sim 2 \times 10^{33} \mathrm{~cm}^{-2} \mathrm{sec}^{-1}$. To utilize the boost in integrated luminosity, key CLEO sub-detectors have been designed and installed to reduce physics backgrounds present in many CLEO analyses. CLEO III contains a new silicon tracking device, a new drift chamber and a novel dedicated particle identification device which replaces a time-of-flight counter. The trigger and data acquisition systems are upgraded to accommodate the higher data taking rate. There are minor modifications to the rest of the CLEO sub-detectors.

\section{CESR Improvements}

To increase the threshold for longitudinal beam instability, a past limitation to higher luminosity, and to provide adequate power to the larger amperage beams, CESR has replaced completely all of its room temperature 5-cell copper cavities in its RF system by 4 single cell superconducting cavities. These new cavities are designed to produce an accelerating gradient of $10 \mathrm{MV} / \mathrm{m}$, allowing the bunch length in CESR to be reduced to $13 \mathrm{~mm}$, to transmit $325 \mathrm{~kW}$ to the beam, and to raise the threshold for longitudinal instability above 1 A total beam current.

Installation of new interaction region (IR) optics is scheduled for the Spring of 2000 to reduce the amplitude of the vertical betatron function $\beta$ at the 
interaction point (IP) to $\beta_{v}^{\star}=13 \mathrm{~mm}$. These optics are two pairs of a set of magnets consisting of a permanent quadrupole magnet and two superconducting (SC) quadrupoles. Each permanent neodymium iron boron magnet provides vertical focusing and is positioned just $337 \mathrm{~mm}$ from the IP. Most of the focusing is provided by the four identical SC magnets that can produce gradients up to $48.4 \mathrm{~T} / \mathrm{m}$ at $1225 \mathrm{~A}$. The entire set of IR optics is contained within $\pm 2.55 \mathrm{~m}$ of the IP. The proximity of the IR optics to the IP necessitates the installation of a new tracking system for CLEO since these new magnets intrude into the volume of CLEO's former configuration.

After the upgrade, CESR will collide beams of 9 nine trains each, with each train composed of 5 bunches separated by 14 ns. The peak total beam current will be $1 \mathrm{~A}$. Opposing beams will continue to circulate in a pretzel orbit scheme and will collide at the IP with a small horizontal crossing angle $\theta_{C}=2.7 \mathrm{mrad}$. The expected luminosity is $L \sim 2 \times 10^{33} \mathrm{~cm}^{-2} \mathrm{sec}^{-1}$.

\section{Particle Identification}

A fast ring imaging Cherenkov detector (RICH) is CLEO's technology choice for particle identification (PID). The general scheme is shown in figure 1 where a charged hadron exits the IP, traverses a solid LiF radiator and produces Cherenkov photons in the familiar cone pattern. The Cherenkov cone expands in an uninstrumented volume outside the radiator before being intercepted by a thin multiwire proportional chamber (MWPC) filled with a photon conversion gas of triethylamine (TEA) and methane. The photoelectrons are amplified near the MWPC anode wires and the resultant charge is then capacitively coupled to cathode pads at the rear of the MWPC which are then read out by front end electronics. The Cherenkov angle is inferred from the pattern of hit cathode pads. The actual implementation of this scheme is shown in figure 2 where a quasi-cylinder of lithium fluoride $(\mathrm{LiF})$ is surrounded at slightly larger radius by a quasi-cylinder of multi-wire proportional counters (MWPCs) with the same azimuthal symmetry. The device covers $80 \%$ of the solid angle.

The design goal for the RICH detector alone is to provide at least $3 \sigma \pi / K$ Cherenkov angle separation at momentum $p=2.8 \mathrm{GeV} / \mathrm{c}$, the maximum possible for $B$ decay daughters at CLEO. Combining a RICH measurement with a current $2 \sigma \mathrm{d} E / \mathrm{d} x$ measurement from the drift chamber for particle momentum exceeding $p=2.2 \mathrm{GeV} / \mathrm{c}$, the overall CLEO design goal is to provide $>3.6 \sigma \pi / K$ separation at high momentum. This corresponds to a Cherenkov angle resolution of $4 \mathrm{mrad}$ per charged track, equivalent to an angular resolution of $14 \mathrm{mrad} /$ photoelectron and 12 photoelectrons per track.

The radiator material is lithium fluoride $(\mathrm{LiF})$ in the form of tiles with planar 


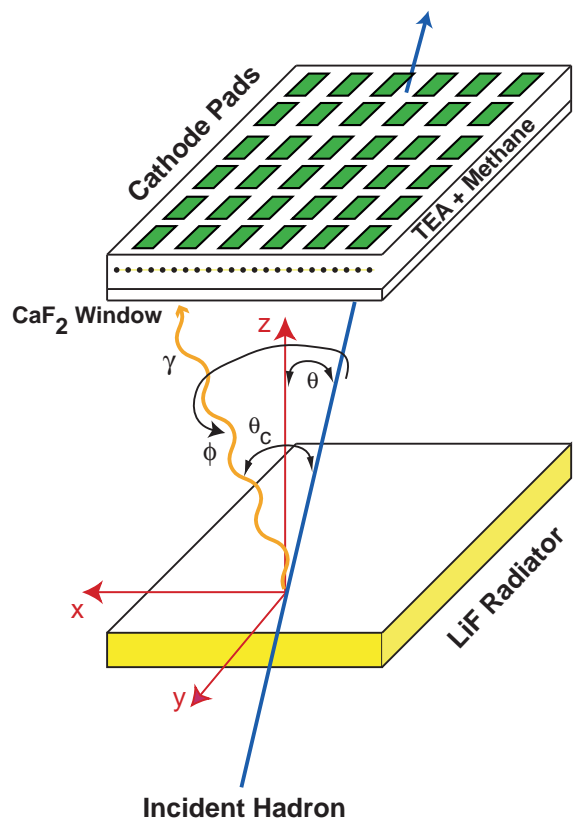

Fig. 1. The CLEO III RICH scheme. See text for explanation.

dimensions $17 \mathrm{~cm} \times 17.5 \mathrm{~cm}$ and mean thickness $10 \mathrm{~mm}$. The tiles are arrayed in a regular pattern to form a quasi-cylinder of $1.6 \mathrm{~m}$ diameter and 30-fold azimuthal symmetry. LiF is selected to accommodate the narrow wavelength $\lambda$ region (135 $\mathrm{nm}<\lambda<165 \mathrm{~nm}$ ) where the TEA-methane quantum efficiency is usable, to minimize the total radiation thickness in front of CLEO's calorimeter, and to minimize the chromatic variation in the radiator index of refraction $n(\lambda)$, which directly affects the Cherenkov angle resolution.

The detected Cherenkov photon patterns form images in the MWPCs of conic sections distorted by refractive effects. Roughly half the Cherenkov cone is trapped inside a radiator crystal by total internal reflection (TIR). Indeed, if all radiator crystals were parallelepipeds, then charged tracks traveling at angle $\theta=90^{\circ}$ with respect to the beamline would have all of their Cherenkov photons trapped inside a radiator crystal by TIR. To prevent this, all radiator crystals within $\theta=90^{\circ} \pm 22^{\circ}$ of the beamline have their top surface cut in a sawtooth pattern[1] so that Cherenkov photons can strike the upper crystal surface at incidence angles below the critical value for TIR.

A cross-sectional view of one of the 30 MWPCs is shown in the upper portion of figure 2. Each MWPC has a $250 \times 20 \mathrm{~cm}^{2}$ rectangular footprint with 70 $\mathrm{Au}$-plated tungsten anode wires of $20 \mu \mathrm{m}$ diameter running longitudinally. Its $2 \mathrm{~mm}$ thick front window is built from 8 rectangular $\left(30 \times 19 \mathrm{~cm}^{2}\right) \mathrm{CaF}_{2}$ crystals that have $100 \mu \mathrm{m} \mathrm{Ag}$ silver traces applied to them. The MWPC cathode is a printed circuit board with $7.5 \times 8 \mathrm{~mm}^{2} \mathrm{Au}$-plated $\mathrm{Cu}$ pads. The MWPC is run at a typical gain $g \sim 4 \times 10^{4}$.

The RICH front end readout electronics is based on a dedicated 64-channel 


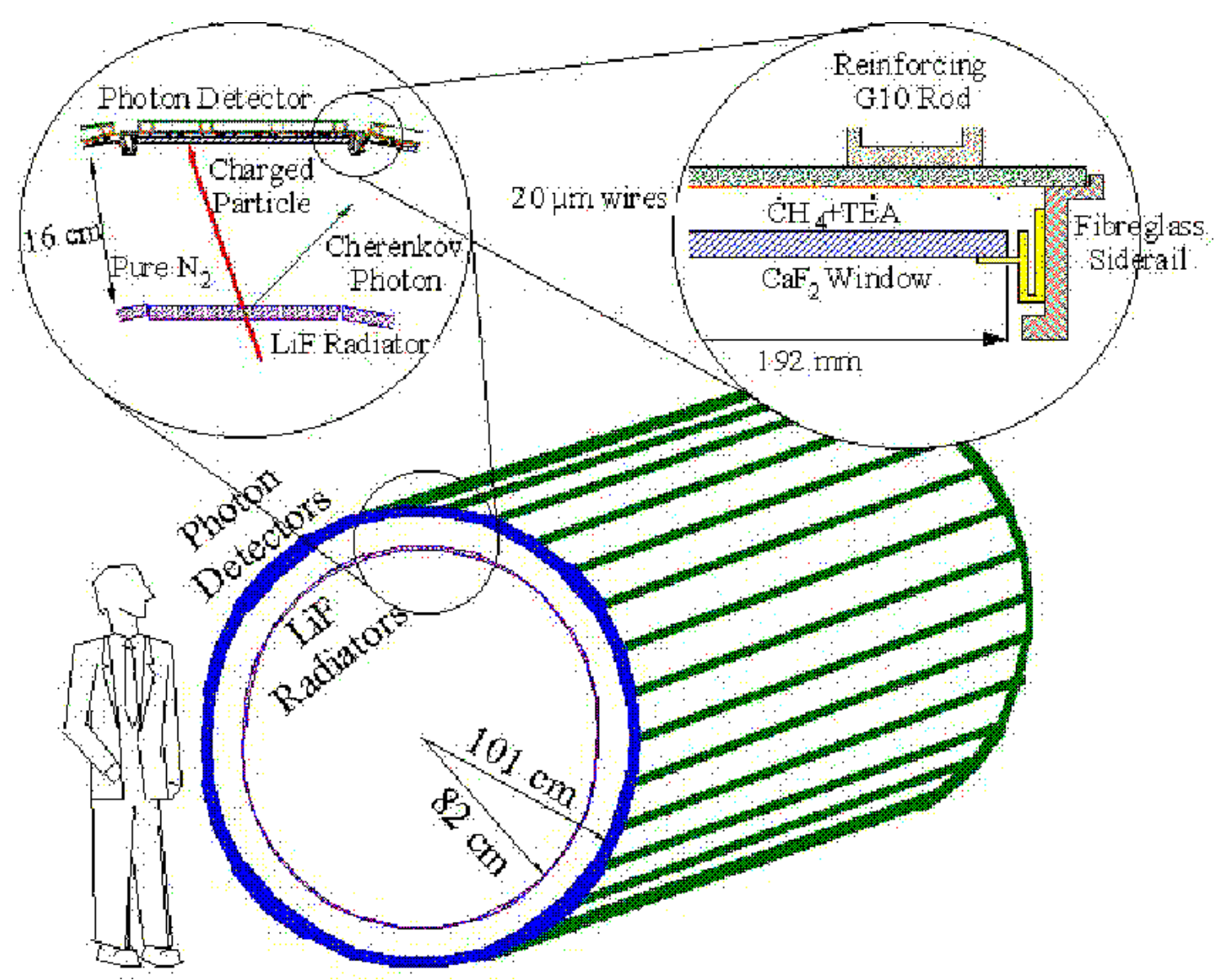

Fig. 2. The key elements of the CLEO III RICH detector. A quasi-cylinder of LiF radiator tiles is nested inside another one, with identical azimuthal symmetry, comprised of MWPCs. Detail of an MWPC is shown in the top half of the figure.

VLSI chip comprised of a preamplifier, a shaper with a programmable peaking time, sample and hold circuitry, and an analog output multiplexer. Through a via, each of the total 230,400 MWPC pads is connected to a preamplifier input channel. The low noise front end has a linear response for input signals up to $\sim 3 \times 10^{5}$ electrons with a measured equivalent noise charge ENC, for detector capacitance $C, \mathrm{ENC}=130 e^{-}+\left(9 e^{-} / \mathrm{pF}\right) C \sim 150 e^{-}$. The front end output is a differential current transmitted serially to off-detector data acquisition boards.

A test beam [2] performed with $100 \mathrm{Gev}$ muons incident on planar and sawtooth radiator crystals imaged by 2 MWPCs yields, for the sawtooth crystal, a mean number of photoelectrons per track $N_{P E}=13.5$, a Cherenkov angle resolution per photoelectron $\sigma_{P E}=10.2 \mathrm{mrad}$, and an overall Cherenkov angle resolution per track $\sigma_{T R K}=4.5 \mathrm{mrad}$. Similar results are obtained for the planar crystal. For the CLEO detector itself, with has improved tracking and larger acceptance, we expect $\sigma_{T R K}=2.9 \mathrm{mrad}$, satisfying our design goal. 


\section{Central Drift Chamber}

The CLEO III central wire tracker is a 47 layer drift chamber with wire layers extending radially from $132 \mathrm{~mm}$ to $790 \mathrm{~mm}$. Outer cathode strips at $797 \mathrm{~mm}$ radius provide a final z-position measurement. The first 16 wire layers are axial and the remaining 31 layers are small angle $(\simeq 25 \mathrm{mrad})$ stereo. Tungsten sense wires are $20 \mu \mathrm{m}$ diameter and the aluminum field wires are $110 \mu \mathrm{m}$ diameter. With a 3:1 field:sense wire ratio, the 9796 drift cells are nearly square with $\simeq 14 \mathrm{~mm}$ width. The average chamber radiation thickness $X / X_{0}=3.3 \%$ for normally incident particles. The drift gas is a 60:40 mixture of helium-propane, selected for its long radiation length $\left(X_{0}>569 \mathrm{~m}\right)$, and its favorable drift and ionization properties.

The mechanical structure of the chamber accommodates the close positioning of the final focus quadrupoles by having a stepped endplate to hold and position the axial wire layers. See figure 3. A conical endplate holds and positions the stereo wires. The wire tension is resisted solely by outer skins that hold the cathode strips. An inner tube provides a gas seal only.

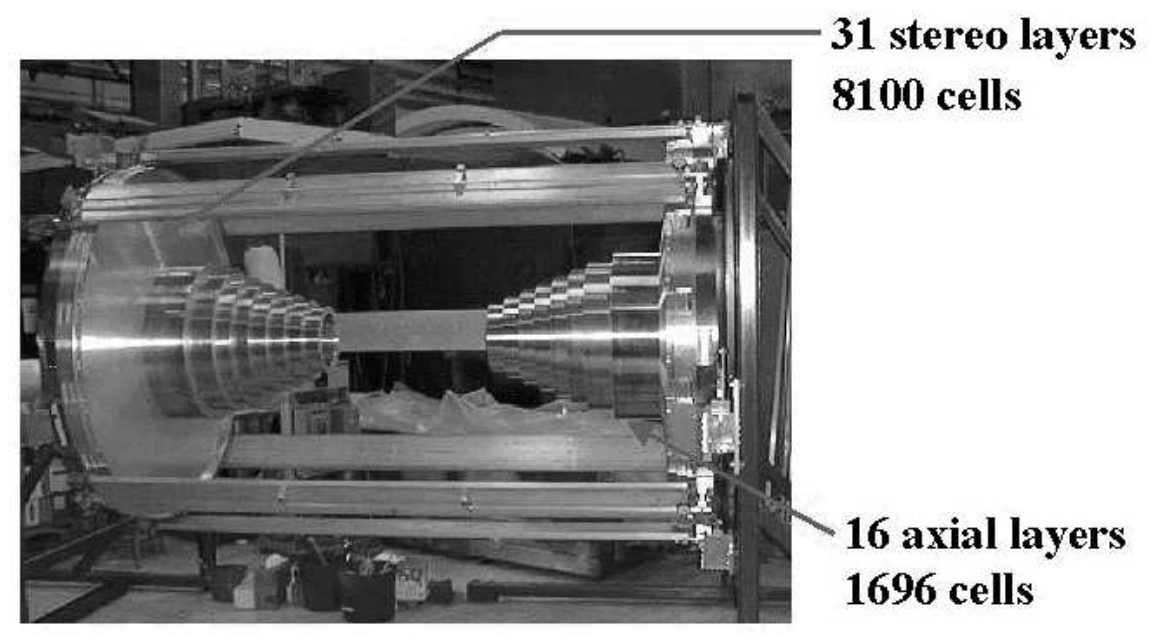

Fig. 3. The CLEO III drift chamber under construction. Note the stepped portion of the endplate used to accommodate the CESR final focus quadrupoles. The number of drift cells is also indicated.

Sense wire signals are read by preamplifiers that drive differential analog current signals to special purpose "time-charge-trigger" (TQT) front end boards that provide signals for timing, charge measurement and the trigger. Signals received by the TQT boards are split into parallel timing/charge and trigger paths. The boards have independent thresholds for the discriminated timing and trigger signals to permit a low trigger rate without degrading timing resolution. The output of the timing and charge-to-time circuits are multiplexed onto the same channel of a LeCroy 1877S Fastbus multi-hit TDC. The dis- 
criminated trigger signal is sent to the trigger system.

\section{Silicon Vertex Detector}

CLEO III includes a 4-layer barrel-style silicon strip vertex detector with double-sided sensor layers located at radii between $2.5 \mathrm{~cm}$ and $10.1 \mathrm{~cm}$ and covering $93 \%$ of the solid angle. To simplify overall ladder production, all 447 of the $300 \mu \mathrm{m}$ thick sensors are identical and contain no coupling capacitors or bias resistors. Ladder lengths span $16 \mathrm{~cm}$ at the inner radius to $53.3 \mathrm{~cm}$ at the outer radius. The n-strips measure the $r-\phi$ coordinate and have a $50 \mu \mathrm{m}$ pitch, while the p-strips measure the z-coordinate and have a $100 \mu \mathrm{m}$ pitch. Considerable care has been taken to minimize sensor capacitance[3]. Approximately 125, 000 total strips are read out.

The vertex detector mechanical design minimizes material in the fiducial region by placing the detector's major mechanical support structure and readout electronics outside this volume. Ladders are mechanically stiffened by gluing V-shaped CVD diamond beams to them and are supported at their ends by copper end cones through kinematic mounts. A flexible kapton circuit electrically connects the sensors to the front end electronics mounted on $\mathrm{BeO}$ hybrids that are attached directly to the copper cones for efficient cooling. A thin carbon fiber tube joins the opposite copper end cones.

A signal-to-noise ratio $S / N>15: 1$ for all sensor layers is the overall detector readout goal. The front end electronics is built from 3 different chips mounted on double-side hybrids. For detector biasing and decoupling, a special 128 channel $R / C$ chip is used with $R=150 \mathrm{M} \Omega$ and $C=150 \mathrm{pF}$. Sensor signals are amplified and shaped by a 128 channel multi-stage preampli-

fier/shaper[4] with a $125 \mathrm{fF}$ feedback capacitor, $200 \mathrm{mV} / \mathrm{mip}$ gain, and a $2 \mu \mathrm{s}$ shaping time. The circuit includes a variable gain stage to accommodate the range in ladder capacitance due to different ladder lengths. The measured equivalent noise charge ENC for the preamplifier/shaper is, for sensor capacitance $C, E N C \sim 150 e^{-}+\left(5.5 e^{-} / \mathrm{pF}\right) C$. The final front end chip is a 128 channel "SVX_CLEO" digitizer/sparsifier based on the FNAL/LBL SVX_II(b)[5]. This chip incorporates a comparator and an 8 bit ADC for each channel. The digitized results can then be sparsified using a chip-wide threshold before being readout serially by an off-detector VME-based data board. 


\section{Trigger System}

The CLEO III trigger system has a single hardware level with a $2 \mu$ s trigger decision time. Since this is much longer than the $14 \mathrm{~ns}$ bunch-to-bunch-spacing, a trigger pipeline is used with all trigger processing done in $42 \mathrm{~ns}$ steps. The level 1 trigger information originates from just 2 detector subsystems: the central drift chamber and the calorimeter. The tracking trigger is built from an "axial" trigger, derived from hits on the 16 axial layers of the drift chamber, and a "stereo" trigger, derived from hits on the chamber's 31 stereo layers. The calorimeter trigger is derived from energy deposition in the 7800 thalium doped CsI calorimeter crystals.

The relatively small number of axial wires (1696) permits the full set to be examined for all possible hit patterns caused by tracks with transverse momentum $p_{\perp}>200 \mathrm{MeV} / \mathrm{c}$. Up to two hits from a valid track can be missing from each of the inner and outer set of 8 wires. Hit patterns are compared against look-up tables for valid tracks using field programmable gate arrays (FPGAs) and up to two hits are allowed to be missing from each of the inner and outer set of 8 wires. Additional circuitry generates track count and topological information to correlate axial and stereo tracks.

The relatively large number of stereo wires (8384) does not make the wire-bywire hit examination used for the axial layers practical. Instead, stereo wires are grouped into $4 \times 4$ arrays and then patterns of hit arrays are compared against those for valid tracks via FPGA lookup logic. Not all arrays along an arc need be hit to satisfy the logic which accommodates tracks with transverse momentum $p_{\perp}>250 \mathrm{MeV} / \mathrm{c}$.

The calorimeter trigger has been substantially upgraded to increase its efficiency, particularly at low energies. Previously, the light output from 16 crystals was summed and shaped, and then compared against low- and high-level energy thresholds. However, energy deposited in the calorimeter associated with a track is typically shared among several crystals. If these crystals lie on the border between adjacent groups of 16 crystals, then it is possible for the separate summed signals to fall below one or both of the thresholds. To eliminate this effect, additional 'tiling' circuitry is used to sum sets of 16 crystals into $2 \times 2$ arrays or 'tiles' of 64 crystals who total signal is then compared against three thresholds. The tiles overlap so that the problem of energy shar-

ing between crystals is eliminated. Other circuitry is used to properly rescale, if necessary, the apparent transverse shower size produced by the tiling circuitry. 


\section{Conclusion}

The superconducting RF cavities, the RICH detector and the central drift chamber are all installed, and CESR and CLEO are now conducting an engineering run scheduled from November 1999 to February 2000. The major sub-detector not quite complete is the silicon vertex detector, due for instal-

lation in February 2000, after which physics running commences. The final focus quadrupoles for CESR are due in Spring 2000 and their installation is the final element of the complete CLEO/CESR upgrade.

\section{Acknowledgments}

The generous assistance of K. Ecklund, V. Fadeyev, R. Mountain and M. Selen in preparing this talk is appreciated.

\section{References}

[1] A. Efimov et al., Nucl. Instr. and Meth. A 365 (1995) 285.

[2] M. Artuso et al., hep-ex/9910054.

[3] I. Shipsey et al., Nucl. Instr. and Meth. A 386 (1997) 37.

[4] H. Kagan et al., Nucl. Instr. and Meth. A 383 (1996) 189.

[5] O. Milgromme et al., Proc. 2nd Intl. Conf. on Dev. and App. of Semiconductor Tracking Detectors, Hiroshima (1995). 